\title{
THERMO-MECHANICAL ANALYSIS OF A SILICON CARBIDE HONEYCOMB COMPONENT APPLIED AS AN ABSORBER FOR CONCENTRATED SOLAR RADIATION
}

Thomas Fend ${ }^{1}$, Peter Schwarzboezl ${ }^{1}$, Olena Smirnova ${ }^{1}$, Martin Schmuecker, ${ }^{2}$ Ferdinand Flucht $^{2}$, Sven Dathe ${ }^{1}$

${ }^{1}$ German Aerospace Center, Institute of Solar Research, DLR, 51147 Koeln, Linder Hoehe, Germany

${ }^{2}$ German Aerospace Center, Institute of Materials Research, DLR, 51147 Koeln, Linder Hoehe, Germany

\section{ABSTRACT}

The study focuses on a numerical model describing the thermo-mechanical processes in an open volumetric solar absorber. In this application thermal loads of up to $850 \mathrm{~kW} / \mathrm{m}^{2}$ induce mechanical stresses inside the volume of the component. The objective of the study is to identify critical thermal load cases which may especially occur during start-up or shut down cases as well as during the transition of clouds. The study can be subdivided into three major parts. In a first step, the mechanical strength of the investigated materials has been determined in double-ring, three or four-point bending tests. In a subsequent numerical stress analysis the maximum stresses in the walls of the honeycomb structure at fracture load have been determined. The results of this combined experimental/numerical approach have been compared with the strength properties of dense materials. In a second step various transient and stationary load cases were considered and the corresponding temperature distributions were calculated. Thirdly, the thermo-mechanical model was used to determine the stress distributions which have been induced. Special attention was paid on the influence of the macro geometry and cell-density. The results of the study allow important conclusions for the operation of the receiver.

\section{INTRODUCTION}

Thanks to its high application temperature of more than $1300^{\circ} \mathrm{C}$, Silicon Carbide (SiC) is used in solar energy, electro-technical, chemical and automobile industries. However, since this ceramic material is not able to undergo plastic deformations, it is very sensible to thermoshocks. The present study has been carried out to investigate, whether typical thermal load cases, as they occur during the application of $\mathrm{SiC}$ honeycomb structures as volumetric receivers in solar tower technology, lead to thermal shocks with critical stress distributions.

The volumetric solar absorber is the key component of solar tower technology, which uses concentrated solar radiation to generate thermal energy which operates a thermal engine.
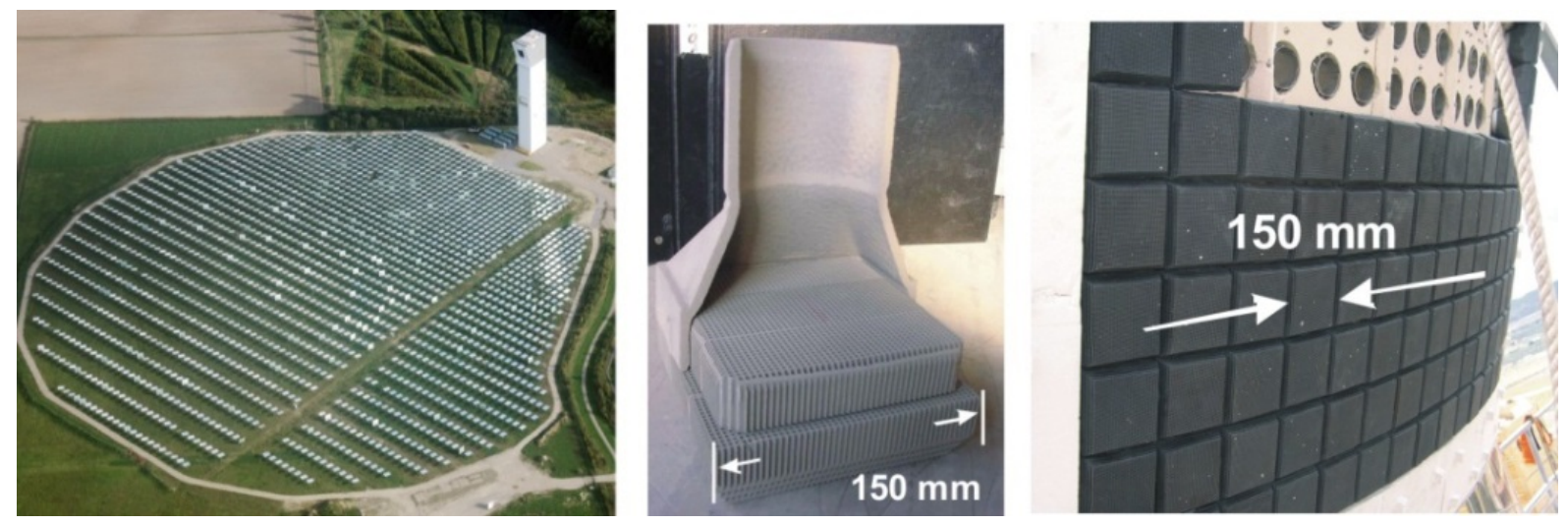

Figure 1. Photographs of the Jülich Solar Tower (left), a part of a single absorber module and the solar receiver during installation 
The concentrated radiation is generated by a large field of individually moving mirrors (heliostats). The focus is located on the top of a tower (Fig. 1, left). The volumetric solar absorber, located in the focus, generally consists of a porous material, which absorbs the radiation and transfers it to air. After that, the air is used to heat up steam in a boiler. The heat transfer in the solar absorber has been investigated already since 1985 and since then, numerous studies have been published [1-4]. A comparative review is given in [5]. The investigated material with the honeycomb structure based on the state-of-the-art-technology shown in Fig. 1 (middle and right), which has also been applied in the solar tower in Jülich [6]. It has been manufactured by St. Gobain IndustrieKeramik Rödenthal GmbH.

The operation experience of the solar volumetric absorber in solar tower technology has shown a good reliability of the honeycomb structure. But the influence of various spatial and temporal temperature gradients at the different load cases on the structure stability is not known. Therefore the aim of the presented thermo-mechanical analysis can be divided into three parts: 1.The determination of the mechanical fracture stress for different geometries of the solar absorber; 2.The determination of the maximum mechanical stress and its localization at the studied load cases and 3.The influence of structure changes on the thermo-mechanical stability for various load cases.

Two macro geometries and two structures with different cell-densities of the volumetric solar absorber have been investigated in the current study. The first one is simple rectangular block geometry (simple geometry) and the second one is a similar one with an additional truncated pyramid in the air outlet zone (real geometry). In the model, the simple geometry was directly drawn in ANSYS 15; the second geometry was made in Inventor 14 and imported in ANSYS afterwards. Both geometries are shown in Fig. 2.

The simple geometry was investigated with a cell-density of $80 \mathrm{cpsi}^{1 *}$. The real geometry was investigated with a cell-density of 80cpsi (Fig. 3 "standard geometry") and with a cell-density of 200cpsi (Fig. 3 “advanced geometry”).

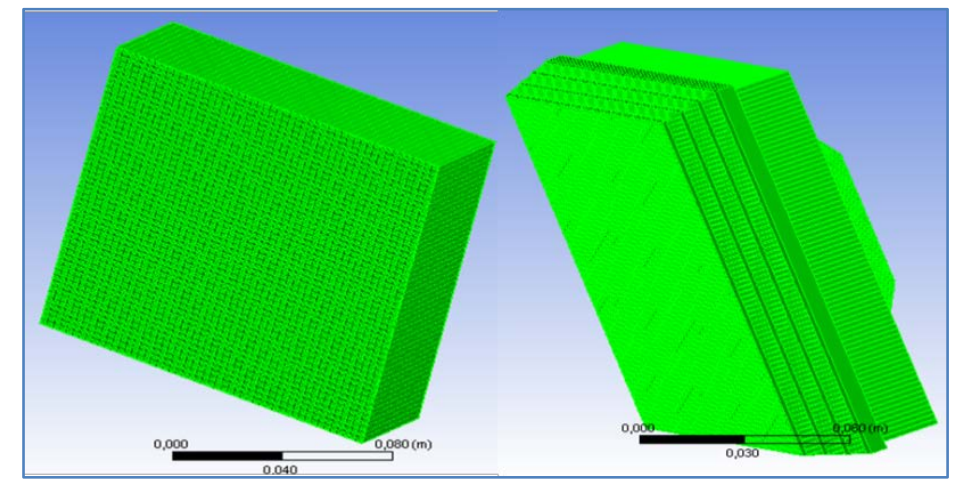

Figure 2. The simple and real geometries of the solar absorber

The various geometries shown above were numerically investigated for different thermal load cases, which are deduced from the practical operation of the component in real service conditions. The numerical results of the thermo-mechanical analysis were compared with the mechanical fracture stress, which was found from bending tests. The values of the fracture force, which were measured experimentally, were then used in the numerical models as the boundary condition for the determination of the fracture stress.

\footnotetext{
${ }^{1}$ channels per square inch
} 


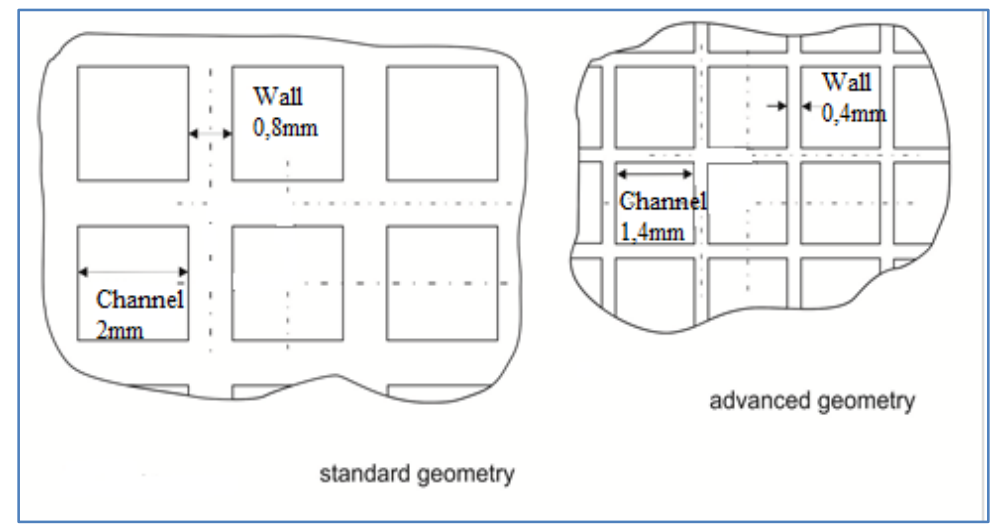

Figure 3. Cross-sectional view of the absorber with cell density 80cpsi (standard geometry) and 200cpsi (advanced geometry)

The numerical work was carried out in two steps using the two different ANSYS modules: thermal and mechanical for each of the studied thermal load cases. The obtained results allowed estimating both the critical temperature differences and the localization of the critical and overcritical mechanical stresses for each of the investigated structures.

The estimation of the maximum thermo-mechanical stress in the porous structure during the critical thermal loads was one of the main aims of the represented investigations. Therefore the following load cases commonly encountered during operation in real service conditions have been investigated:

- Stationary homogenous radiation of app. $650 \mathrm{~kW} / \mathrm{m}^{2}$

- Stationary homogenous radiation of app. $650 \mathrm{~kW} / \mathrm{m}^{2}$ with an additional nonhomogeneous temperature distribution in diagonal or axial directions

- Stationary non-homogeneous radiation

- Transient cooling and heating at homogenous time-dependent radiation (cloud-effect)

\section{METHODOLOGY}

Experimental and numerical estimation of the fracture stress

The fracture stress is used as the main criterion for the assessment of the solar absorber stability. Three kinds of standard bending tests have been carried out to estimate this quantity: double ring and 3- and 4- points bending tests [7]. The test set-ups and theoretical equations for the determination of the maximum stress in the dense material are shown in Fig.4.

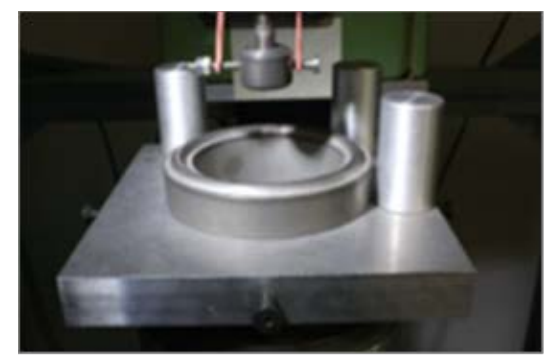

double ring bending test (1)

$$
\sigma=1.04 \cdot \frac{F_{\max }}{h^{2}}
$$

[MPa]

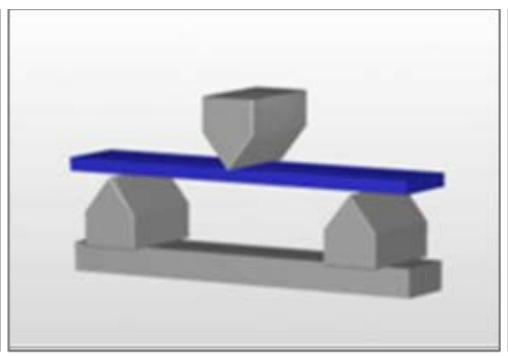

3-point bending test (2) $\sigma=1.5 \cdot \frac{F_{\max } \cdot L_{1}}{b \cdot h^{2}}[\mathrm{MPa}]$

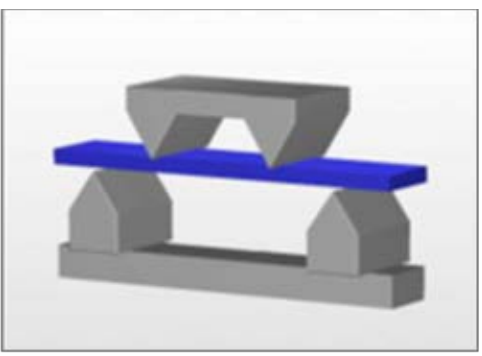

4-point bending test (3) $\sigma=1.5 \cdot \frac{F_{\max } \cdot\left(L_{1}-L_{2}\right)}{b \cdot h^{2}}[\mathrm{MPa}]$

Figure 4. Schematic drawing of the bending tests and theoretical equations for the calculation of the maximum stress in a dense material. 
The tests were made for the two honeycomb structures with cell densities 80cpsi and 200cpsi. The numeric models simulated both the dense body and the honeycomb structures for the estimation of the maximum stress in the wall of the cellular structure. The validation of the numeric models was realised by the comparison between the simulated and calculated data for the dense body.

\section{Numerical Models}

Each numerical model can be divided into two parts. The first is the numerical simulation of the bending test with the mechanical module and the second one is the thermomechanical model, which consists of two modules: thermal and mechanical. The models are 3-D models. The numerical model was calculated for the four load cases and the three geometries described in the introduction. The bending test was simulated for the dense body and for the honeycomb structure. The sample geometry as well as the dimensions of the test equipment and the fracture force was taken from the experiments.

The thermo-mechanical model was studied in two modules. The temperature distribution was calculated in a thermal module and then it was imported into the mechanical module, where the resulting stress distribution was obtained. The transient tasks were considered in three steps:

- The stationary thermal module, which calculated the initial temperature distribution for the transient task

- The transient thermal module for the time dependent temperature distribution

- The stationary mechanical module for the stress distribution at selected points of time

Boundary and domain conditions of the models for the investigated load cases

The boundary conditions of the models can be divided into two groups. The main conditions, which have used in all load cases, belong to the first group. The specific conditions, each of which used only in some of the load cases, belong to the second group. The main boundary conditions of the thermal modules are: the inlet solar radiation and forced convective heat transfer in the channels of the solar absorber. The solar radiation was described as a homogeneous or non-homogeneous heat flow density on the front surface. The homogeneous heat flow density was $q_{0}=650 \mathrm{~kW} / \mathrm{m}^{2}$ (normal incidence). A non-normal incident angle $(\varphi)$ of the radiation was taken into account for the sloped front surface (in the real structure):

$$
q_{s l .}=q_{0} \cdot \sin (\varphi)
$$

The non-homogeneous heat flow density was described with Gaussian function (5) or according to the measured temperature distribution as a temperature boundary condition.

$$
q=q_{0} \cdot e^{-k \cdot\left(\left[x-\frac{x_{\max }}{N} \cdot L_{x}\right]^{2}+\left[y-\frac{y_{\max }}{N} \cdot L_{y}\right]^{2}\right)}
$$

Here, $N$ is the number of cells on the front surface, $k$ is a measure for the non-homogeneity, $x_{\max }, y_{\max }$ are the coordinates in $\mathrm{x}$ and in y directions, $L_{x}=L_{y}=0,14[\mathrm{~m}]$ is the dimension of the front surface.

The convective boundary conditions in the channel were determined with a previous COMSOL model [8] in dependence of the channel size. The corresponding diagrams and equations for the simple geometry with cell density 80cpsi are shown in Fig. 5 and in equations (6) and (7). 
$\alpha=35-2,95 \cdot 10^{6} \cdot z^{3}-2,2 \cdot 10^{5} \cdot z^{2}-5,2 \cdot 10^{3} \cdot z$

$\mathrm{T}_{\text {air }}=332,15-3,050728 \cdot 10^{7} \cdot z^{3}-2,60648 \cdot 10^{6} \cdot z^{2}-7,3595 \cdot 10^{4} \cdot z$

The convective heat transfer depends on the absorber geometry, therefore each of the studied structures has own equations.

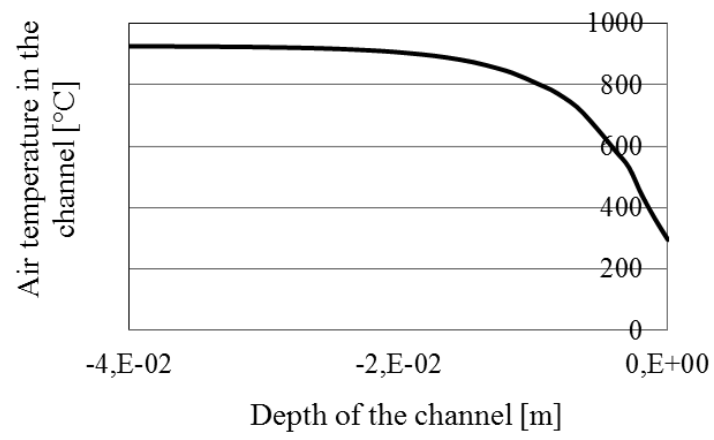

(a)

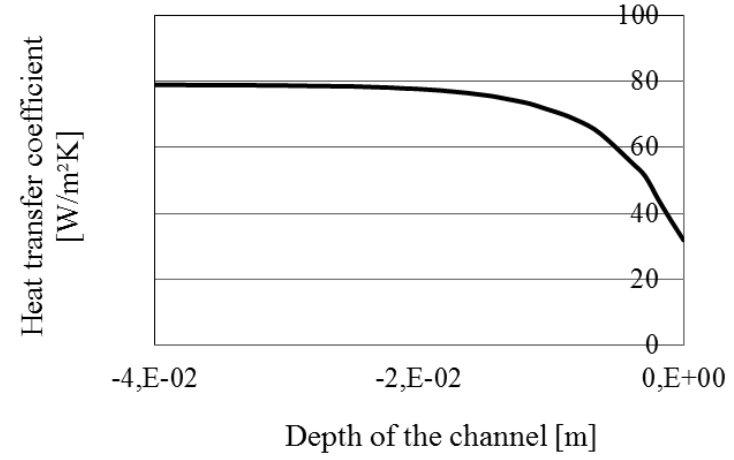

(b)

Figure 5. The air temperature in the channel (a) and the heat transfer coefficient (b) from one channel model COMSOL 3.5 for the standard channel $(2: 0,8 \mathrm{~mm})$

The air temperatures in the channels for the load cases of the cloud-effect have been estimated as $15^{\circ} \mathrm{C}$ lower compared to the ones described with the equation above.

To describe the transient intensity of the solar radiation during a cloud transition, a transient heat flow was used as a specific boundary condition:

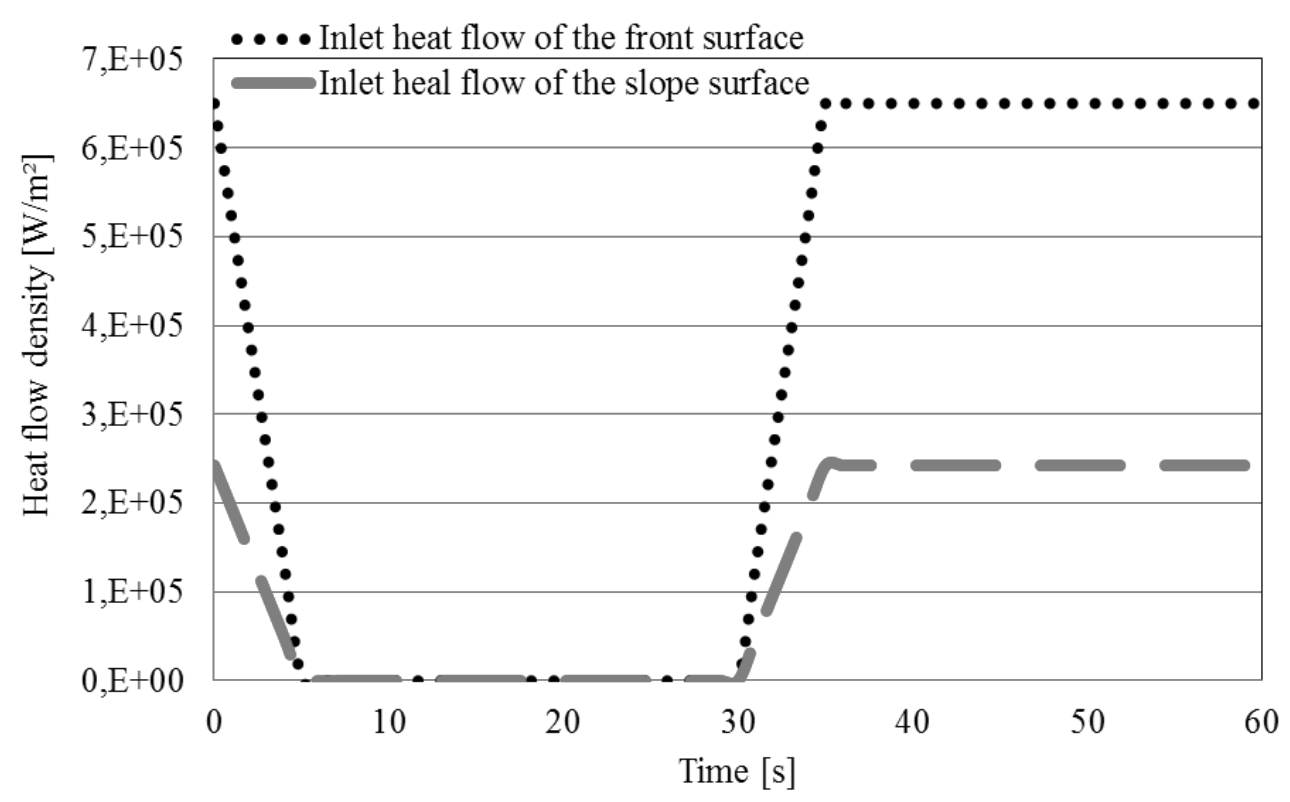

Figure 6. The inlet heat flow density for the simulation cloud - effect

The time-dependent decrease and increase of the heat flow was assumed to last 5 seconds (Fig. 6). This short time has been considered to be the maximum possible thermal load, which represents a very fast transition of a cloud. The following equations describe the timedependent heat flow:

$$
q_{0}(t)=q_{0}-130000 \cdot t
$$




$$
q_{S l .}=q_{0} \cdot \sin (\varphi)-48488 \cdot t
$$

A homogeneous heat flow was assumed as the first boundary condition to obtain the initial temperature distribution for all studied structures.

Two additional temperature boundary conditions have been used in two specific load cases: a temperature gradient in $\mathrm{x}$ direction and a "diagonal" boundary temperature with two different temperatures at the 4 lateral boundaries of the receiver module with different temperatures at opposite boundaries:

$$
\begin{array}{ll}
\mathrm{T}=873+1428.5 \cdot \mathrm{x}[\mathrm{K}], & \mathrm{T}=1073-1428.5 \cdot \mathrm{x}[\mathrm{K}] \\
T=873[\mathrm{~K}], & \mathrm{T}=1073[\mathrm{~K}]
\end{array}
$$

\section{RESULTS AND DISCUSSIONS}

The results of the bending test simulation were used for the estimation of the maximum mechanical stresses in the real micro geometry of the material, which do not come out of the bending theory. This allows us to estimate whether the thermal loads are below or above critical values. The study of the influence of the geometry on the maximum stress allows an estimation of the reliability of the investigated structures.

The results of the bending test simulation

The simulation of the bending test enables a comparison between the fractures stress in the real geometry of the material and the fracture stress in a virtual "dense" material, which is delivered by the fracture force of the experiments and bending theory (equations in Fig. 4). Additionally and for a validation of the model, the fracture stress of a corresponding dense material was modeled. The fracture stress results for the dense body are summarized in Table 1. The comparison gives a good correspondence between the theoretical and simulated data for the dense body with the difference $\approx 7-8 \%$.

Table 1. The theoretical and simulated fracture stress for the dense body

\begin{tabular}{|l|c|c|c|c|c|c|c|c|}
\hline & \multicolumn{3}{|c|}{ Double ring flexural test } & \multicolumn{3}{c|}{ 3-Points flex. test } & \multicolumn{2}{c|}{ 4-Points flex. test } \\
\cline { 2 - 9 } & 1.Test & 2.Test & 3.Test & 1.Test & 2.Test & 3.Test & 1.Test & 2.Test \\
\hline $\begin{array}{l}\text { Theoretic fracture } \\
\text { stress [MPa] }\end{array}$ & 24 & 21 & 22 & 31 & 46 & 25 & 22 & 9 \\
\hline $\begin{array}{l}\text { Simulated } \\
\text { fracture stress } \\
{[\mathrm{MPa}}\end{array}$ & 22 & 19 & 20 & 28 & 42 & 23 & 20 & 9 \\
\hline
\end{tabular}

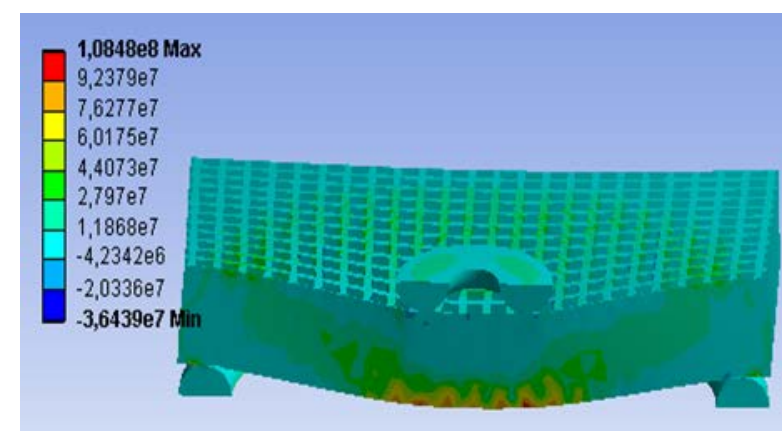

(a)

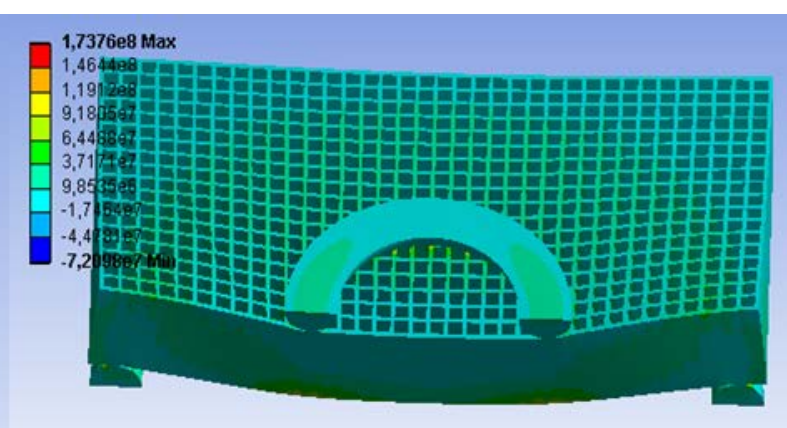

(b)

Figure 7. Maximum stress in the principal coordinate system for the double ring bend test for the 80cpsi (a) and for the 200cpsi sample (b) 
The simulation results of the double ring bending tests with the fracture stress for two studied honeycomb structures are shown in Fig.7. The comparison of the fracture parameters can be found in Table 2. The comparison of these results for the honeycomb structure shows, that the stress in the real geometry is about a factor of 5 (80 cpsi) and 11 (200 cpsi) higher than for the corresponding dense material.

Table 2. Comparison of the fracture parameters for the investigated structures

\begin{tabular}{|c|c|c|c|c|c|}
\hline \multirow{2}{*}{$\begin{array}{c}\text { Porosity } \\
\text { [cpsi] }\end{array}$} & \multicolumn{2}{|c|}{ Experimental data } & \multicolumn{2}{c|}{ Numerical data } \\
\cline { 3 - 6 } & $\begin{array}{c}\text { thickness } \\
\mathrm{h}[\mathrm{mm}]\end{array}$ & $\begin{array}{c}\text { break force } \\
\mathrm{F}_{\max }[\mathrm{N}]\end{array}$ & $\begin{array}{c}\text { calculated break } \\
\text { stress } \sigma[\mathrm{MPa}]\end{array}$ & $\begin{array}{c}\text { simulated break } \\
\text { stress } \sigma \text { [MPa] }\end{array}$ \\
\hline \multirow{2}{*}{80} & solid body & 9.36 & 1839 & 22 & 20 \\
\cline { 2 - 6 } & $\begin{array}{c}\text { honeycomb } \\
\text { structure }\end{array}$ & 9.36 & 1839 & - & 108 \\
\hline 200 & solid body & 8.67 & 1026 & 14 & 16 \\
\cline { 2 - 6 } & $\begin{array}{c}\text { honeycomb } \\
\text { structure }\end{array}$ & 8.67 & 1026 & - & 174 \\
\hline
\end{tabular}

The results of the thermo-mechanical models

The thermo-mechanical analysis of the studied solar absorber was made on the basis of the comparison between the different structures at an equal load case.

Load case: Stationary homogeneous radiation

This load case was considered for the simple structure with cell density 80cpsi and for the real structure with both cell densities $80 \mathrm{cpsi}$ and 200cpsi. The temperature distributions at stationary homogeneous radiation are given in Fig.8.

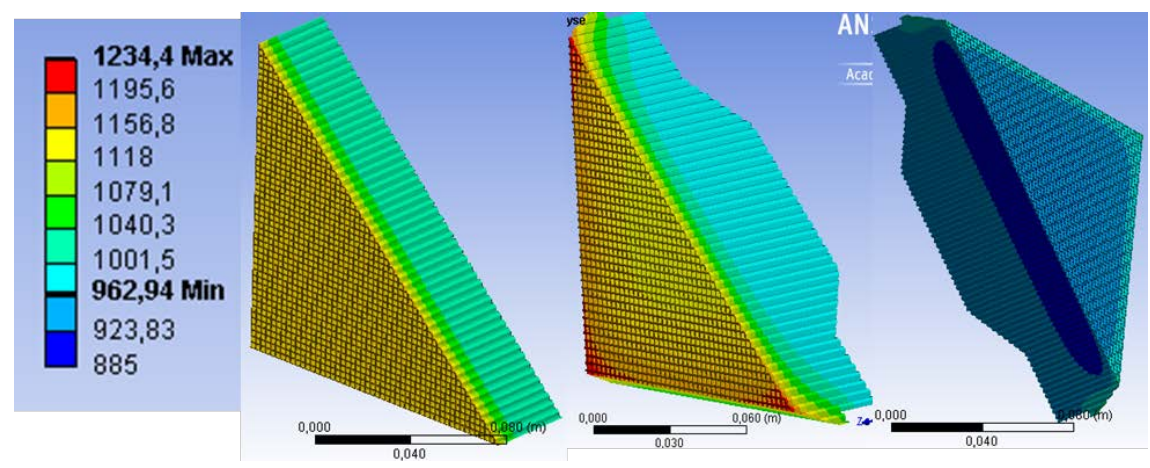

Figure 8. The temperature fields at stationary homogeneous radiation from left to right: simple geometry, real geometry 80cpsi and real geometry 200cpsi.

Values up to $1234 \mathrm{~K}$ for the real geometry (80cpsi) and up to $1043 \mathrm{~K}$ for the real geometry (200cpsi) are reached. The lower temperature with the 200 cpsi structure can be explained by the larger heat transfer surface.

As expected, the temperature distributions have a layer character for all structures. This character is homogeneous for the simple structure and non-homogeneous for the real structure. The inhomogeneous temperature distribution can be explained by the influence of the truncated pyramid. The front temperature increases at the corners and decreases in the center of the front surface. This effect is more pronounced for the $200 \mathrm{cpsi}$ absorber. The temperature field in the absorber with the porosity 200cpsi shows an unexpected decline in the depth $\approx 13-16 \mathrm{~mm}$. The temperature increase behind this minimum may be explained by a conductive heat flow in the solid from the outer regions. 

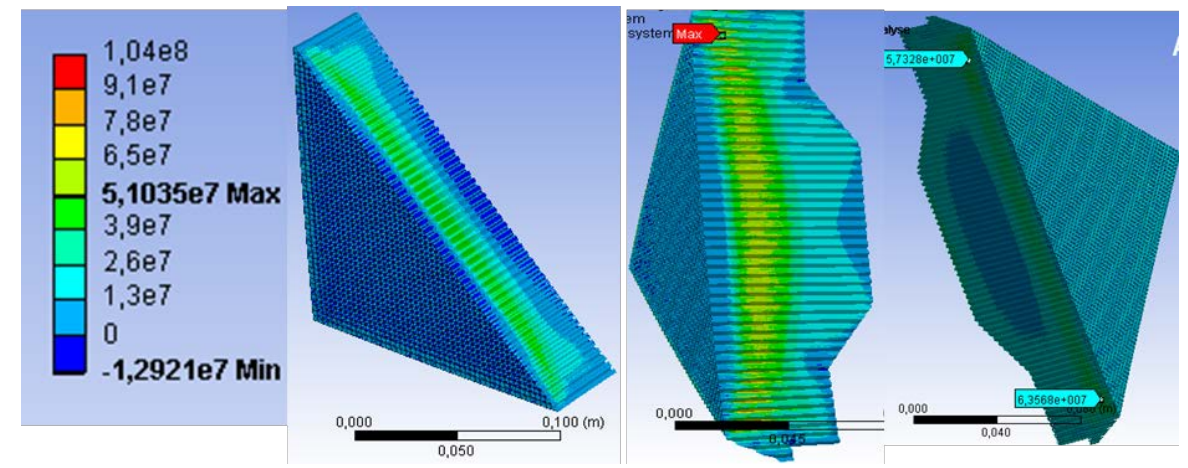

Figure 9. Maximum stress in the absorber (from left to right) with simple geometry (80 cpsi), with real geometry (80cpsi and 200cpsi).

The maximum stress distributions for the investigated load case are summarized in Fig. 9. The maximum stress in the absorber with simple geometry is located in $16-18 \mathrm{~mm}$ distance from the irradiated surface and achieves 51MPa. The maximum stress for both real geometries is locates in $10-12 \mathrm{~mm}$ distance from the irradiated surface and achieves $104 \mathrm{MPa}$ for the coarse structure and 66MPa for the finer structure. The largest stress in the 80cpsi real structure is directly due to the largest temperature difference in this sample.

Results at non-homogeneous radiation/non homogeneous temperature boundary conditions

Fig. 10 shows the temperature and the stress distributions for the load case stationary non-homogeneous radiation. The density of the incoming inlet heat flow due to concentrated solar radiation ranges from $850 \mathrm{~kW} / \mathrm{m}^{2}$ to $1.57 \mathrm{MW} / \mathrm{m}^{2}$. The corresponding peak temperature measured temperature reaches $1309 \mathrm{~K}$. The simulated maximum stress achieves $97 \mathrm{MPa}$ at a temperature difference of $650 \mathrm{~K}$.

The distributions of temperature and mechanical stress for the load case with stationary homogeneous radiation and an additional temperature difference at the boundaries are shown in Fig.11. Both of these extreme load cases show overcritical mechanical stresses of up to $142.2 \mathrm{MPa}$ in the diagonal direction and up to 208.4MPa in the axial direction.

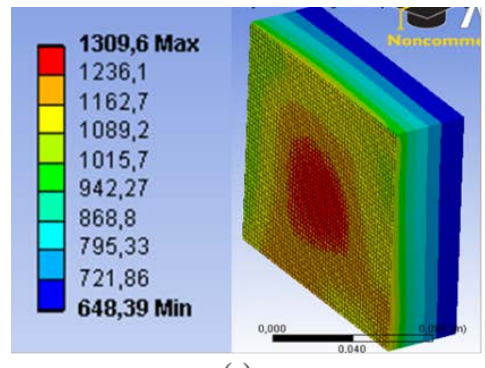

(a)

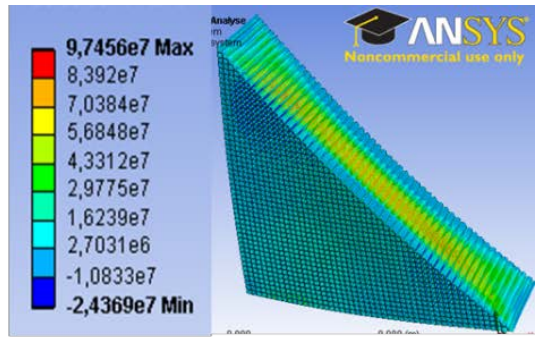

(b)

Figure 10. Temperature (a) and stress distribution (b) at the non-homogeneous radiation 


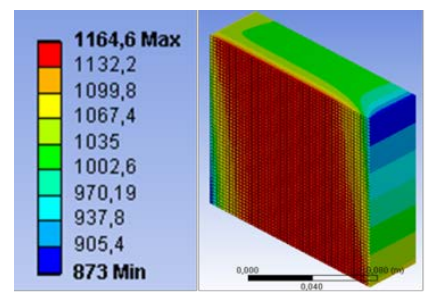

(a)

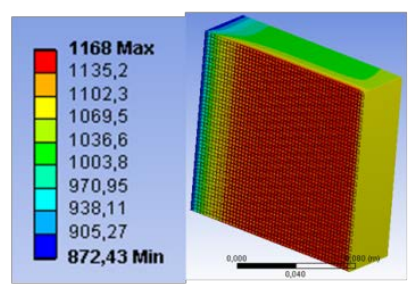

(c)

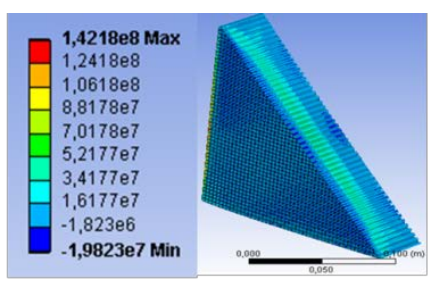

(b)

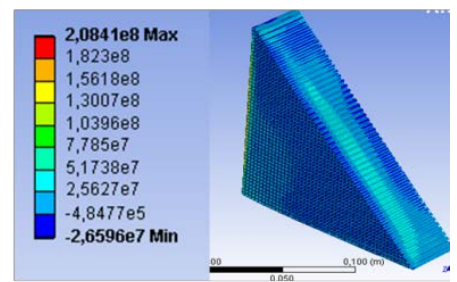

(d)

Figure 11. Temperature distributions at stationary homogeneous radiation with additional temperature gradients in the diagonal (a) and in the axial direction (c). (b) and (d) show corresponding stresses.

The results of the "cloud effect" case

The cloud-effect is modeled as a time dependent cooling and heating processes. It uses a time dependent homogeneous heat flux shown in Fig.6. The time dependent temperature distributions of the maximum and minimal temperatures for both studied real structures are shown in Fig.12.

The comparison yields larger values for the coarser structure. The minimal temperature difference is 5 seconds and the maximum one 30 seconds after cooling start. The values are for the finer structure $\Delta T_{\max }=195^{\circ} \mathrm{C}$ and $\Delta T_{\min }=116^{\circ} \mathrm{C}$, for the coarser structure they are $\Delta T_{\max }=273^{\circ} \mathrm{C} ; \Delta T_{\min }=65^{\circ} \mathrm{C}$.

The values of the mechanical stress have been taken at the starting time and at the end of the process, and in four intermediate points during the cooling and the heating. Figure 13 shows the change of the temperature fields at these points.

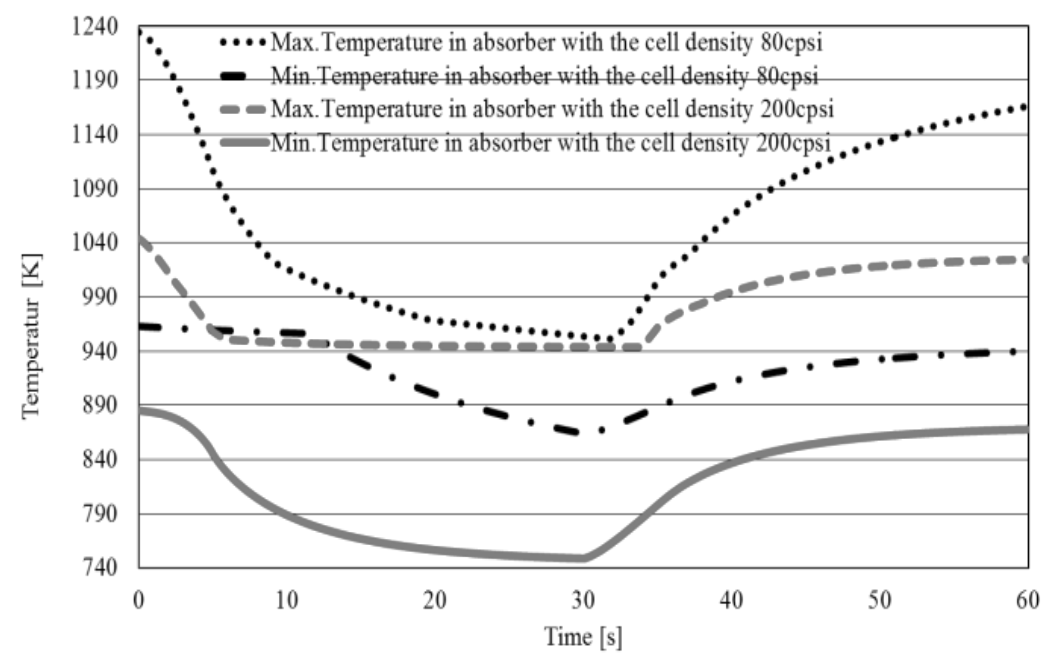

Figure12. The comparison of the maximum and minimal temperatures for both real structures 


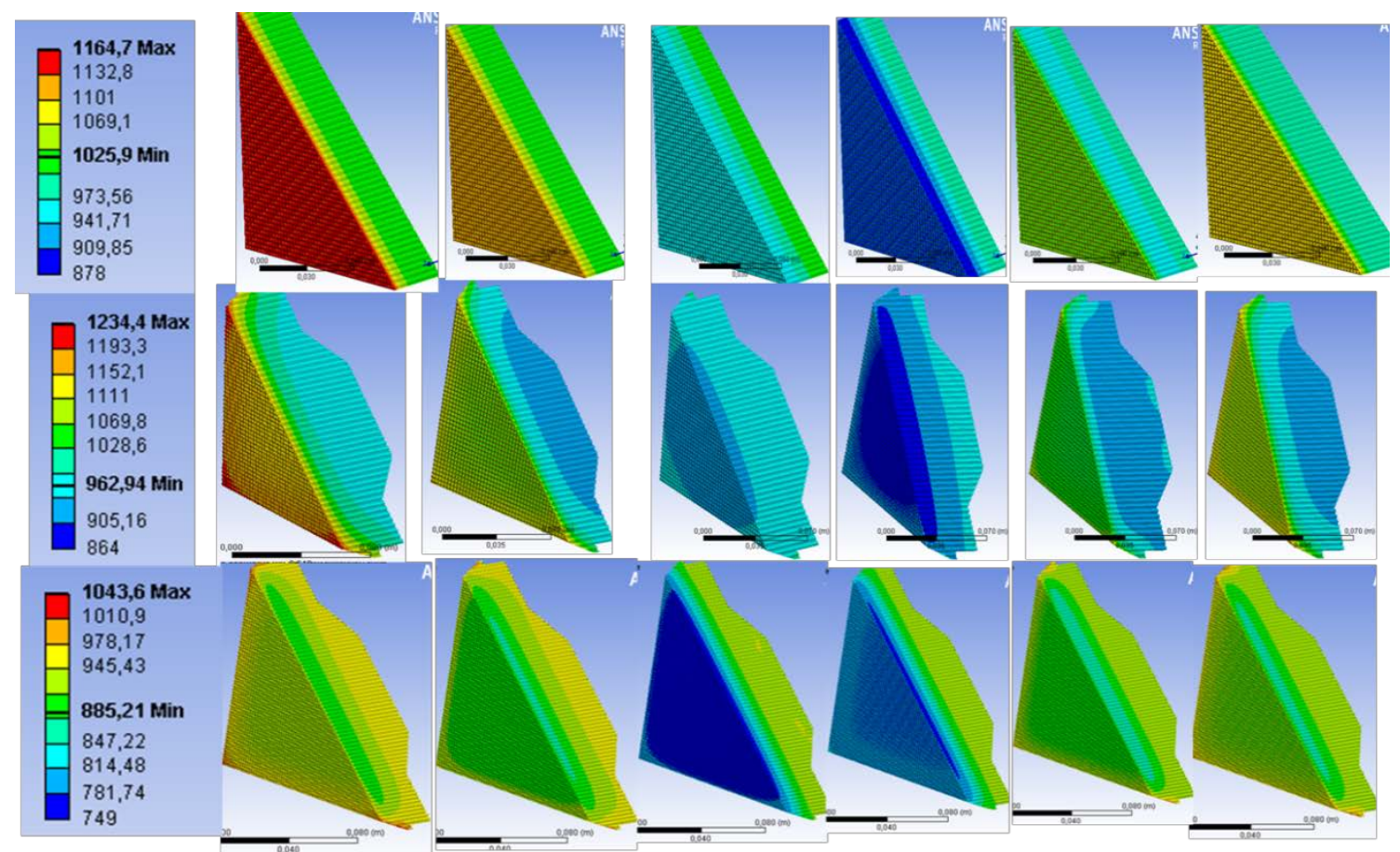

Figure 13. The temperature fields in the following time points from left to right: at start, 3s after the cooling start, $15 \mathrm{~s}$ after the cooling start, $3 \mathrm{~s}$ after the heating start, $15 \mathrm{~s}$ after the heating start and at the end (30s after the heating start). The figures of the upper series belong to the simple geometry, the figures of the middle series to the real geometry with 80cpsi and the lower series to the real geometry with 200cpsi.

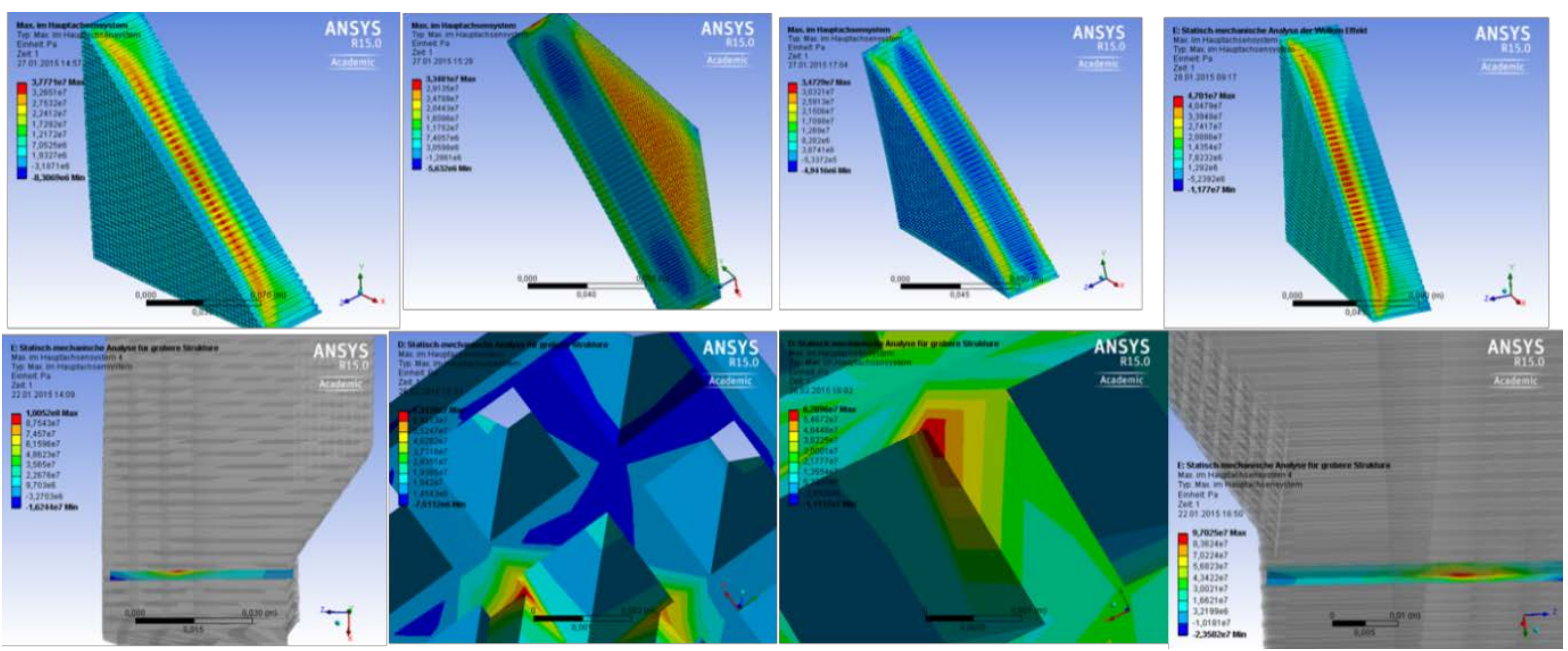

Figure 14. The stress distribution in the simple structure (upper line) and the increase of the maximum mechanical stress locations in the real geometry 80cpsi of the solar absorber at the 4 intermediate time points mentioned above (from left to right).

The distribution of the corresponding stresses is shown in Fig. 14. Together with the analysis of Fig. 15 the results of the cloud effect model can be summarized as follows:

- During the cooling period the point of maximum stress moves from a distance of 16$18 \mathrm{~mm}$ from the irradiated surface to the air outlet surface. During the heating period it moves back for the load case with the simple structure and with the cell-density 80cpsi (the upper pictures in Fig.14)

- In the case of the pyramid geometry the point of maximum stress is located at channel walls in $15 \mathrm{~mm}$ distance from the front surface at the edges of the truncated pyramid. During the cooling process it moves to the air outlet surface of these channels and 
during the heating it moves back. This is true for the load case with the real structure (80cpsi, the bottom pictures in Fig.14)

- $\quad$ The 200cpsi real structure behaves similar as the 80 cpsi one, only with slightly larger stress values

Finally, the fracture stress of the double ring bending test was compared with the maximum mechanical stress of the cloud-effect. The result is shown in Fig.15 (left). It becomes clear, that the maximum mechanical stress of the sample with the simple structure is lower than fracture stress. The maximum mechanical stress of the sample with the standard real structure (cell density 80cpsi) achieves the critical value at the start and at the end of the process. The maximum stress is higher than the fracture stress for the finer real structure in the middle of the process. The dependence between of the maximum mechanical stress and maximum temperature difference is especially important for the thermo-mechanical analysis. It is shown in Fig.15 (right). As expected, the dependence is almost linear for each of the studied structures. It may be also stated, that the cooling process is more critical than the heating process for all studied structures. The critical and over critical values of the mechanical stress correspond to temperature differences of app. $230^{\circ} \mathrm{C}$ for the standard structure and $130^{\circ} \mathrm{C}$ for the finer structure.
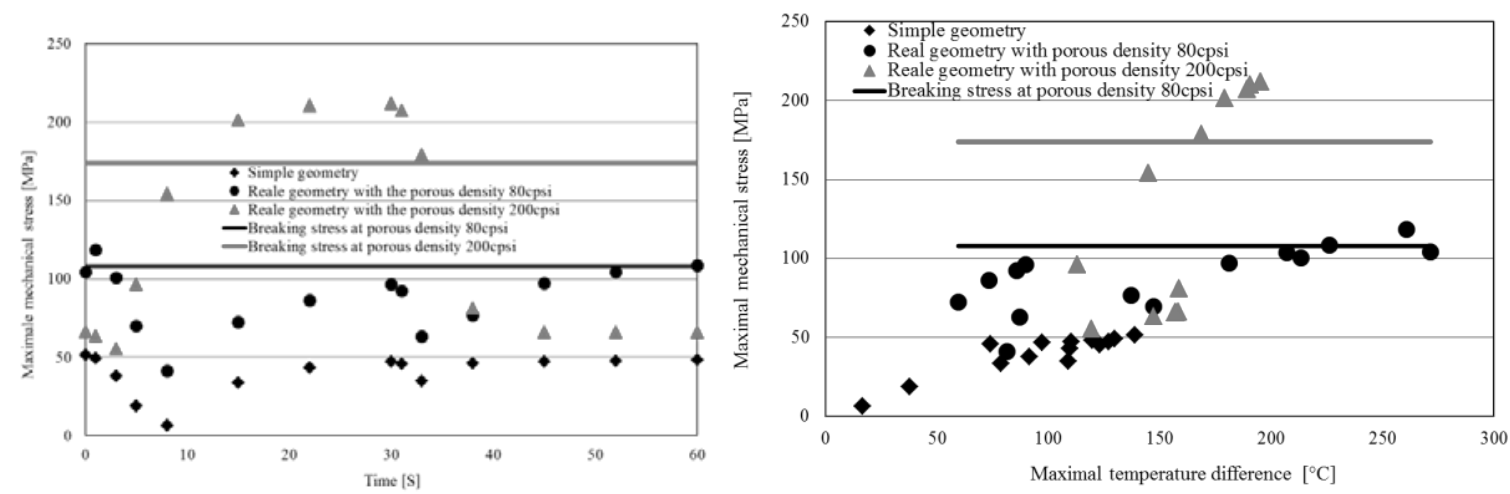

Figure15. Comparison between the fracture stress and the maximum mechanical stress during the cloud-effect simulation (left) and the maximum mechanical stress as a function of the maximum temperature difference (right).

\section{CONCLUSIONS}

The following conclusions can be made according to the represented thermomechanical analysis:

1. The thermo-mechanical models presented allow an investigation of the influence of representative stationary and transient load cases on the stresses in a solar receiver.

2. The fracture stress determined from the bending test simulation can be used to assess the amount of mechanical stress occurring during representative load cases

3. During a cloud transition, the point of maximum mechanical stress moves from a localization in the middle of the receiver to the outlet surface (cooling phase) and back during the heating phase.

4. The comparison of the two macro geometries shows that for the truncated pyramid case the temperature difference and maximum stress is significantly larger.

5. The maximum mechanical stress in the sample with the finer structure is always larger than in the one with the coarse structure at the equal thermal load.

6. Both the transient thermal load and the inhomogeneous boundary conditions increase the maximum mechanical stress.

7. Although the finer structure of the solar absorber shows a higher thermal effectiveness, the coarser one promises a safer operation and a larger reliability. 


\section{REFERENCES:}

[1] H. Fricker, Bull. SEV/VSE, 76, pp. 10-16

[2] M. Romero, R. Buck, J. E. Pacheco, An Update on Solar Central Receiver Systems, Projects, and Technologies. Journal of Solar Energy Engineering124, 98-108 (2002)

[3] A. Kribus, R. Zaibel, D. Carrey, A. Segal, J. Karni, A solar - driven combined cycle power plant, Solar Energy Vol. 62, No 2, 1998, pp.121-129

[4] M. Becker, Th. Fend, B. Hoffschmidt, R. Pitz-Paal, Theoretical and numerical investigation of flow stability in porous materials applied as volumetric solar receivers, 1 Solar Energy 80 (2006) p.1241 - 1248

[5] Th. Fend, B. Hoffschmidt, R. Pitz-Paal, O. Reutter, Chapter: "Cellular ceramics use in solar radiation conversion", from the book M. Scheffler and P. Colombo: "Cellular ceramics: structure, manufacturing and applications", Willey - VCH GmbH \& Co. KgA, Weinheim, 2005

[6] Th. Fend, B. Hoffschmidt, R. Pitz-Paal, O. Reutter, P. Rietbrock, Porous materials as open volumetric solar receivers experimental determination of thermophysical and heat transfer properties, Energy 29 (2004), (5-6), pp.823 - 833

[7] Metallic materials - Bend test (ISO 7438:2005); German version EN ISO 7438:2005

[8] Th. Fend, P. Schwarzbözl, O. Smirnova, D. Schöllgen, Ch. Jakob (2013) Numerical Investigation of Flow and Heat Transfer in a Volumetric Solar Receiver. Renewable Energy, 60, Seiten 655-661. ISSN 0960-1481 